A N N A L E S Annales de Bretagne et des Pays de l'Ouest

\title{
Simon, Gilles, Plogoff, l'apprentissage de la mobilisation sociale
}

\section{Maël Garrin}

\section{(2) OpenEdition}

1 Journals

\section{Édition électronique}

URL : http://journals.openedition.org/abpo/2044

DOI : $10.4000 / a b p o .2044$

ISSN : 2108-6443

\section{Éditeur}

Presses universitaires de Rennes

\section{Édition imprimée}

Date de publication : 30 mai 2011

Pagination : 193-194

ISBN : 978-2-7535-1691-5

ISSN : 0399-0826

\section{Référence électronique}

Maël Garrin, «Simon, Gilles, Plogoff, I'apprentissage de la mobilisation sociale », Annales de Bretagne et des Pays de l'Ouest [En ligne], 118-2 | 2011, mis en ligne le 30 juin 2013, consulté le 23 septembre 2020. URL : http://journals.openedition.org/abpo/2044 ; DOI : https://doi.org/10.4000/abpo.2044

Ce document a été généré automatiquement le 23 septembre 2020.

(c) Presses universitaires de Rennes 


\title{
Simon, Gilles, Plogoff, l'apprentissage de la mobilisation sociale
}

\author{
Maël Garrin
}

\section{RÉFÉRENCE}

SIMON, Gilles, Plogoff, l'apprentissage de la mobilisation sociale, Rennes, PUR, coll.

« Histoire », 2010, 412 p.

1 La mobilisation antinucléaire de Plogoff fait aujourd'hui figure de mythe pour qui s'intéresse un peu au militantisme écologiste, et, même au-delà de ces milieux, a durablement marqué les esprits. Avec le recul nécessaire, un peu moins de 30 ans après son achèvement, Gilles Simon se penche sur le sujet en publiant, la version éditoriale de sa thèse de sciences politiques soutenue à Rennes en 2008.

2 Afin d'analyser les différentes étapes de la mobilisation, il a mené une large enquête en rencontrant de nombreux protagonistes des événements, tant du côté des militants que des journalistes, des élus ou des promoteurs de «l'atome civil», réalisant au total 56 entretiens. Le dépouillement intégral des journaux de l'époque, qu'il s'agisse de la presse quotidienne régionale, des nouvelles revues écologistes ou de la presse nationale, et la compilation de nombreuses autres sources (tracts, affiches, documentation technique officielle, etc.) viennent également apporter de nombreux éléments à sa réflexion. Chacune de ses analyses est méticuleusement confrontée à des concepts théoriques développés dans les nombreux ouvrages d'histoire, de sociologie et de sciences politiques auxquels il fait référence.

3 Plogoff est resté dans les mémoires comme étant le lieu où s'est cristallisée la mobilisation antinucléaire. Si le titre du livre de Gilles Simon, qui évoque cette commune du cap Sizun, peut sembler restrictif, l'auteur s'intéresse pourtant, dans une première moitié du texte, à un ensemble d'«aires de mobilisation" antinucléaire, 
réparties en Basse Bretagne, dont il justifie la cohérence par leurs nombreuses interactions. En effet, avant de devenir celle de «Plogoff » à la fin de l'année 1978 lors du choix définitif du lieu d'implantation d'une centrale nucléaire en Bretagne par les pouvoir publics, la mobilisation antinucléaire dans cette région s'est ancrée dans un certain nombre d'autres lieux. Les principaux d'entre eux, en termes de densité de l'activité militante, avaient pour point central un des cinq sites pré-retenus par EDF pour l'implantation d'une centrale, faisant suite à l'annonce du programme nucléaire français en 1974.

4 S'il choisit d'étudier la mobilisation antinucléaire en Basse-Bretagne, ce n'est pas uniquement pour revenir sur les faits en eux-mêmes, mais pour jeter une lumière nouvelle sur ceux-ci en développant la thèse selon laquelle toute mobilisation sociale génère de nombreuses formes d'apprentissage. Dans le cas de «Plogoff », les acteurs de la mobilisation n'étaient pas des militants-nés. Parmi ces acteurs, d'origine particulièrement hétérogène, l'auteur distingue deux principales composantes. La première, comprenant les écologistes politiques du Cap Sizun ou d'ailleurs, avait déjà une certaine "expérience de l'engagement militant", mais qui ne la préparait pas entièrement aux divers rebondissements du combat antinucléaire. La seconde, celle des Plogoffites - souvent retraités de la Marine ou femmes de marins au commerce - était encore plus novice en la matière. Au cours de leurs huit années de mobilisation, jusqu'à l'aboutissement heureux qu'ils ont connu en 1981, les nouveaux militants ont donc mis en œuvre un certain nombre de techniques pour se former à de nouveaux "savoirs militants » leur permettant de construire progressivement leur action d'opposition à la centrale nucléaire que l'État projetait d'implanter sur leur commune.

5 Gilles Simon retrace au cours de son étude les différentes étapes de cet apprentissage. Des premiers tâtonnements des CLIN, les Collectifs locaux d'information nucléaire, pour échanger des informations sur la lutte antinucléaire, à la mise en relation autour d'une cause commune d'" agents sociaux» aussi divers que des anciens combattants plogoffites ou des autonomistes morlaisiens en passant par les élus du Cap Sizun et de nombreuses associations et partis politiques, un long chemin a été parcouru. L'apprentissage de la mobilisation antinucléaire, c'est aussi celui de la construction d'un discours cohérent sur le plan scientifique pour faire face aux promoteurs d'EDF, nécessitant un investissement personnel de lecture de documentation technique. Ce discours doit s'accompagner d'une rhétorique militante pour pouvoir toucher une population variée, et donc sensible à des arguments d'ordre divers, pour la convaincre de rejoindre la mobilisation. Cet apprentissage, c'est encore la formation aux techniques du rassemblement des foules sous ses diverses formes, qu'elles soient classiques telles des manifestations, ou plus inventives comme des processions rappelant les pardons traditionnels ou des fêtes antinucléaires, pour faire en sorte que ces instants renforcent la cohésion entre les différents acteurs mobilisés tout en diffusant toujours plus le message antinucléaire. Cet apprentissage, c'est celui du rapport ambigu que les militants se doivent d'avoir aux médias, en tentant par exemple de diffuser leur message aux journalistes locaux tout en sachant que les directions des journaux ne se caractérisent pas par leur position antinucléaire. Cet apprentissage, c'est enfin celui du rapport des militants aux forces de l'ordre, notamment lors d'affrontements qui ont eu lieu pendant l'enquête d'utilité publique de la centrale au début de l'année 1980. Faisant preuve d'une grande inventivité, les Plogoffites essayaient alors chaque jour de trouver de nouvelles formes symboliques pour marquer leur opposition tout en dénonçant la présence disproportionnée des forces de police. Ce 
sont tous ces aspects, et bien d'autres encore, que l'auteur développe longuement au cours de son étude, montrant toute la complexité des interconnexions entre les acteurs à l'origine d'une mobilisation sociale.

6 Un seul point négatif est à souligner à propos des écrits de Gilles Simon : le lecteur tatillon regrettera les trop nombreuses fautes de grammaire qui parsèment le texte. Cela ne diminue en rien la portée de l'étude de l'auteur, qui nous offre une lecture éclairante de la mobilisation antinucléaire en Bretagne entre 1974 et 1981.

INDEX

Thèmes : Plogoff

\section{AUTEURS}

MAËL GARRIN

master 2 HSC - Université Rennes 2 Haute-Bretagne 